\title{
Sweetening the Deal: Glycosylation and its Clinical Applications
}

\section{Yichi Zhang ${ }^{1 *}$ and Lichun Sun ${ }^{1,2,3 *}$}

\author{
${ }^{1}$ Tulane University School of Medicine, Department of Medicine. New Orleans, Louisiana, USA \\ ${ }^{2}$ Shenzhen Academy of Peptide Targeting, Technology at Pingshan and Shenzhen, Tyercan Bio-pharm Co., Ltd., Shenzhen, Guangdong, China \\ ${ }^{3}$ Sino-US Innovative Bio-Medical Centre, and Hunan Beautide Pharmaceuticals, Xiangtan, Hunan, China
}

${ }^{*}$ Corresponding author: Lichun Sun, Department of Medicine, School of Medicine, Tulane University Health Sciences, Centre, New Orleans, USA, Tel: +1-504-988-1179, E-mail: Isun@tulane.edu, Yichi Zhang, Tulane University School of Medicine, Department of Medicine. New Orleans, Louisiana, USA, Tel: +1-202-445-0576; E-mail: yzhang52@tulane.edu

Received date: June 17, 2020; Accepted date: June 18, 2020; Published date: June 30, 2020

Citation: Zhang Y, Sun L (2020) Sweetening the Deal: Glycosylation and its Clinical Applications. J Biomedical Sci Vol.9 No.3:9.

\section{Abstract}

\begin{abstract}
Glycosylation, an important and nearly ubiquitous form of post-translational modification in eukaryotes, has been studied for its clinical applications. Its complex nature and heterogeneity of functions in the human body has allowed researchers to take multiple approaches in applying it to modern medicine. These approaches include glycosylation of pharmaceuticals to improve delivery; using glycosylation as vaccine/drug targets; editing the glycosylation pattern of immune system components; and profiling glycosylation signatures of diseases for diagnostic tests. In addition, glycosylation has also been used as a tool in developing potential therapeutics for the Covid-19 pandemic. This review covers and summarizes recent, interesting findings associated with the various approaches in the clinical study of glycosylation and aims to inspire future research in this promising direction.
\end{abstract}

Keywords: Glycosylation; Medicine; Pharmaceutical; Vaccine; Cancer; Immunology; Covid-19

\section{Overview}

Glycosylation is one of the most common post-translational modifications in eukaryotic protein processing [1]. These reactions are carried out in the endoplasmic reticulum or the Golgi apparatus, where various sugars can be conjugated to proteins via the action of glycosyltransferases.

Among different forms of glycosylation reactions, O-linked and $\mathrm{N}$-linked processes are the most studied and have already shown to be implicated in a wide array of physiologic and pathologic processes. O-linked glycosylation refers to the attachment of glycans to serine $(S)$ or threonine $(T)$ residues of proteins. One of the many significances of this reaction is that it is an essential step in the physiologic formation of proteoglycans, which contain long chained glycosaminoglycans (GAGs) to serve a lubricative purpose in joints and other tissues [2]. Unlike O-linked glycosylation, which typically occurs in the Golgi apparatus, N-linked glycosylation is known to start in the endoplasmic reticulum. It involves the addition of $\mathrm{N}$ acetylglucosamine (GlcNAc) to the asparagine residue side chain. Several other monosaccharide and derivatives could then be added upon this foundation. $\mathrm{N}$-glycans are shown to play important regulatory roles in both intracellular and extracellular signalling [3]. Interestingly, $\mathrm{N}$-linked glycosylation is demonstrated to be a regulator in its own glycosyltransferase activity as well [4]. This may provide further opportunities for researchers to fine-tune glycosylation for specific aims in their studies.

Over the recent decade, the expansion of both breadth and depth of research into glycosylation has brought on newer insights on their structure, function and purpose. Specifically, researchers have dived into various clinical aspects involving glycosylation, with aims to find better therapeutic methods using its unique biochemical characteristics. This review seeks to survey recent advances in the study of the clinical aspects of glycosylation and will be presenting significant findings from multiple angles in the following sections.

\section{Glycosylating Pharmaceuticals for Delivery Improvement}

One of the largest barriers to achieving improved therapeutic effect with modern drugs is how to manipulate pharmacokinetics to illicit the most appropriate pharmacodynamic response from the human body. Over the past few decades, researchers have come up with a cornucopia of ways to enhance the delivery of a drug's active ingredients to the desired human tissue. These methods include but are not limited to: conjugation to proteins, liposomal packaging, PEGylation, and the inhibition of efflux pumps $[5,6]$. Thus, it is not surprising that some researchers turned to glycosylation as another potential method to improving drug delivery. A large assortment of pharmaceuticals has since been designed, studied and produced using glycosylation as a major complementary process. These drugs have been trailed and used to combat many diseases, ranging from various tumours, infectious diseases to autoimmunity. 
Anti-neoplastic are powerful agents used in chemotherapy to combat the growth of cancer. Many classes of anti-cancer drugs have been developed, with a growing focus on how to concentrate the drug within neoplastic tissues. This is due to the wide array of side effects that these chemotherapy agents are often associated with, some of them severe enough to cause damage in any normal tissue they distribute to as well. The concept of the "Magic Bullet" as conceived by Paul Ehrlich has then always become to ultimate pursuit of researchers looking to develop highly specific "Targeted Therapy" for cancer [7].

Glycosylation of anti-neoplastic agents may help further such efforts and contribute to a more specific, effective and safe cancer therapy. For example, Ifosfamide, a widely used alkylating agent for haematological cancers, has been shown to have less toxic effects in vitro when conjugated to glucose. Doxorubicin, an anti-tumour antibiotic, is shown to have increased in vivo efficacy against HepG2 tumour cells when conjugated to 2-amino-2-deoxy-D-glucose [8]. In a separate study, researchers studied how they could improve the bioavailability of Rituximab, a well-known agent used for nonHodgkin's B-lymphomas [9]. Rituximab is normally an OGlcNAcylated protein whose activity can be inhibited by endogenous O-GIcNAcases. Researchers then co-administered Rituximab with Thiamet $\mathrm{G}$, an inhibitor of O-GlcNAcase, which led to increased concentrations of Rituximab, without altering its native functions. See Figure $\mathbf{1}$ for a demonstration of this mechanism regarding Rituximab.

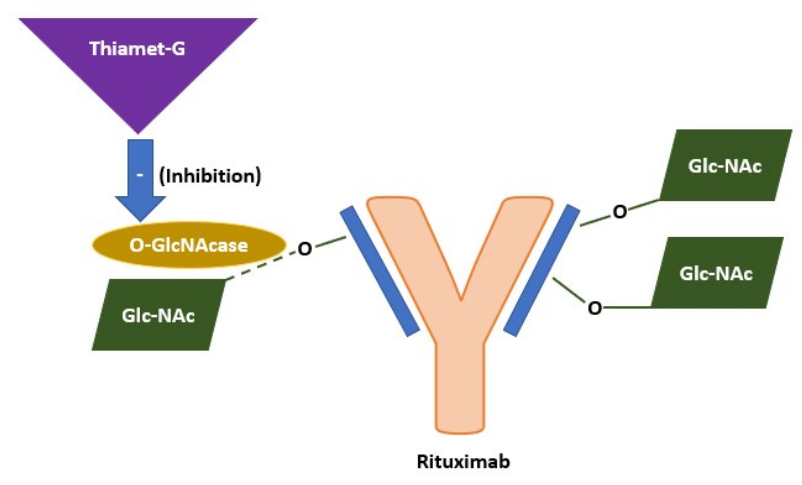

Figure 1: Thiamet-G Inhibition of O-GlcNAcase Affects Rituximab Glycosylation Pattern.

Standard rituximab light chains (Blue) have OGlcNAcylations (Green) at serine 7,12 and 14 catalysed by OGlcNacase (Gold), a glycotransferase. Thiamet G (Purple) inhibits this enzyme and thus changes the glycosylation pattern on Rituximab, improving its production and concentration [9]. Diagram not drawn to actual scale.

A similar finding was discovered with Trastuzumab, a HER2/neu antagonist commonly used for breast cancer [10]. Differential glycosylation patterns on the drug could lead to size and charge heterogeneity and may affect ultimate therapeutic effect. Arruda et al. also found that Zidovudine glycosylation patterns affected its efficacy on melanoma [11].
Thus, to maximize the efficacy and minimize side effects of anti-cancer agents, finding the optimal glycosylation pattern can be a promising start.

In addition to combating cancer, glycosylated drugs have also been studied in their efficacies to combat infectious diseases. The $\mathrm{N}$-glycosylation pattern of Equine Tetherin has been demonstrated to play important role in its subcellular localization, thus altering its antiviral activity [12]. Two antimicrobial peptides, when glycosylated, showed 2-to-16-fold decrease in antimicrobial efficacy against MRSA, VRE and other strains of bacteria [13].

In various other areas of medicine, glycosylation effects on therapeutic agents' efficacy was also observed. Reservatrol, a newly derived stilbene from berries, has shown some neuroprotective efficacy against Alzheimer's and Parkinson's disease. However, this efficacy is largely limited by its low halflife and bioavailability, a problem that researchers hope to solve by changing its glycosylation pattern among other modifications [14]. In the autoimmunity realm, Fc glycosylation of anti-human IFN-alpha2b antibodies has been shown to influence their binding affinity, thus potentially affecting their efficacy in treating Systemic Lupus Erythematous [15]. In haematology, certain glycosylation patterns on Factor XIII-B prolonged its serum half-life, affecting the body's coagulative balance [16]. In endocrinology, Nglycosylation of Calcitonin receptor resulted in increased peptide hormone affinity [17]. If such findings were made for other G-protein coupled receptors as well, it may affect our entire approach to modern hormone replacement therapy.

Thus, controlling the appropriate glycosylation pattern is the key to discovering uncharted territory in modern pharmaceuticals.

\section{Targeting Glycosylation for Vaccine and Drug Development}

In addition to manipulating the glycosylation pattern on drugs, researchers have also found ways to use glycosylation "landmarks" in tissues and pathogens as excellent immunogens and targets for vaccine and drug development.

Aubry et al studied the BclA3 glycosylation pattern of $C$. Difficile spores to develop a conjugate vaccine specific for the spores. Some success was seen in vitro with the Anti-spore serum recognizing the spore-specific glycan moiety. However, animal testing showed that the vaccine did not provide protection against the infection [18]. Despite the results, this study demonstrates the usefulness of glycosylation patterns in vaccine development. Similar work is being performed for $C$. Jejuni, where researchers hope to find the key $\mathrm{O}$ and $\mathrm{N}$-linked glycosylation patterns that grant the bacteria its human virulence and target them with a vaccine [19]. For Hepatitis B, researchers hope to change its surface glycosylation pattern to be more immunogenic, thus paving the way for more effective and longer lasting vaccines [20]. One study even hoped to design the unthinkable, a HIV vaccine by enhancing glycosylation of the envelope via gene editing in Chinese 
Hamster Ovary (CHO) cells [21]. The key challenge to developing the HIV vaccine is the virus' lack of key "glycandependent epitopes" on its envelope. Thus, Li et al are hoping to make certain glycans "stick" to the envelope by inhibiting viral proteolytic processes, thus providing a target for vaccines.

Most modern vaccines are geared towards the prevention of an infectious disease, but with maturing lab techniques to target specific glycosylation patterns, certain novel vaccine types could be possible. For example, Beckwith et al are looking into the possibility of cancer vaccine design. MUC1, a cell-surface mucin, is often glycosylated in an aberrant pattern in carcinomas. These tumour-associated carbohydrate antigens (TACAs) provide a promising target for immunogenicity and vaccine development [22]. Similar work is being performed for breast cancer, one of the most prevalent neoplasms in the world. Researchers are looking into the epithelial-mesenchymal transition (EMT) of breast cancer cells and how it leads to an abnormal glycosylation pattern. This pattern, once fully analyzed, may then provide new approaches in therapeutic design [23]. Some neoplasms, such as Colorectal cancer, expresses an enzyme called glucosylceramide synthase (GCS), a modifier of glycosylation pattern. This enzyme is notorious for contributing to resistance to an array of anti-cancer agents. Thus, researchers used RNA interference to knock-out GCS in the tumour and observed a restoration of its sensitivity for Oxaliplatin, a platinum-based anti-cancer agent [24]. See Figure 2 for a diagram depicting this mechanism.

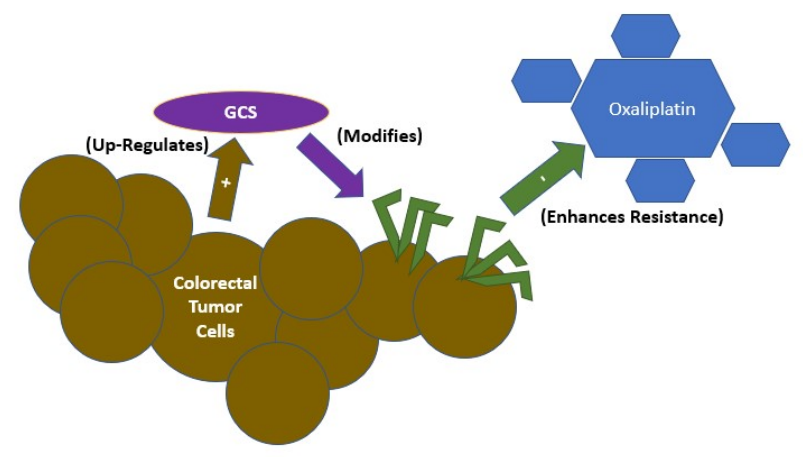

Figure 2: Colorectal Tumour Cells Up-regulate GCS Enhance Resistance to Oxaliplatin.

Colorectal tumour cells (Brown) upregulate glucosylceramide modifying enzyme, GCS (Purple), to change surface glycosylation pattern (Green). This change increases tumour cell resistance towards Oxaliplatin (Blue), a platinumbased chemotherapy [24]. Diagram not drawn to actual scale.

Aside from tumours, glycoprotein pattern recognition has also been studied for its potential use in treating diabetes mellitus and its long-term complications [25]. The abnormal metabolism of glycoproteins and its derivatives contributes greatly to the pathogenesis and progression of diabetes. Understanding how to restore normal glycosylation patterns and glycoprotein metabolism will be of great benefit to the over 350 million people coping with diabetes worldwide.

\section{Modifying Glycosylation for Immunity Refitting}

Often overshadowed by the potent effects of modern pharmaceuticals, the innate and adaptive immune systems of the human body are often the strongest defences against various pathologies. Thus, to activate the full potential of the human immune system in combating disease is a goal of modern clinical research.

Glycosylation of immune system components have been widely observed in the human body, but the effects of these modifications have yet to be fully explored. For example, OGlcNAcylation has been demonstrated to play regulatory roles in the proliferation of $\mathrm{T}$ and $\mathrm{B}$ Lymphocytes. These glycosyl modifications also affect the antiviral response from macrophages. Interestingly, O-GlcNAcylation also promotes neutrophil activity while inhibiting NK cell function [26]. Nlinked glycosylation has its own wide array of effects of cellular signalling as well, affecting antigenic recognition, immune cell activation, proliferation and migration [27]. The immense difficulty in dissecting the specific effects of $\mathrm{O}$ and $\mathrm{N}$-linked glycosylation arises both from the inherent complexity and heterogeneity of the sugar chains as well as the large number of different conjugation sites available on various proteins. However, some interesting findings have been made and provide a promising foundation for the way forward.

Kumpel et al studied the glycosylation patterns on the Fc portion of Anti-D IgG antibodies from 23 different human and rodent cell lines in relation to their clinical effects. They concluded that high galactosylation combined with low fucosylation were the ideal patterns for the antibody, achieving the most effective clearance of red cells at relatively lower doses [28]. In another study, Corsiero et al has shown that $\mathrm{N}$-linked glycosylation in the Fab region of Anti-NET RAAntibodies were largely responsible for their immunoreactivity. Removing the glycans from the antibodies resulted in a $90 \%$ decrease in its reactivity towards citrullinated histone $\mathrm{H} 2 \mathrm{~B}$ [29]. Findings like these may have major implications on future therapies for autoimmune diseases like rheumatoid arthritis.

Furthermore, glycosyl modifications of the immune system also affects the body's response to foreign pathogens. For example, a deficiency in glusaminyl transferase 1 (Gcnt1) will impair the core-2 O-glycan synthesis pathway, increasing the patient's susceptibility towards infection with M. Tuberculosis. This Gcnt1 deficiency was linked with the increased expression of neutrophil attractant CXCL2 in the lungs [30]. In similar fashion, Adherent-Invasive E. coli (AIEC), which has been shown to be central in pathogenesis in certain cases of irritable bowel disease, also interacts with host O-GlcNacs during GI colonization. AIEC infection leads to significant increase in UDP-GIcNAc, which is responsible for the formation of $\mathrm{O}$ GlcNac. Thus, the bacteria can create an amenable environment for itself via modifying the glycosylation pattern 
of the host. See Figure $\mathbf{3}$ for a demonstration of AIEC's enhanced colonization tactic involving glycosylation.

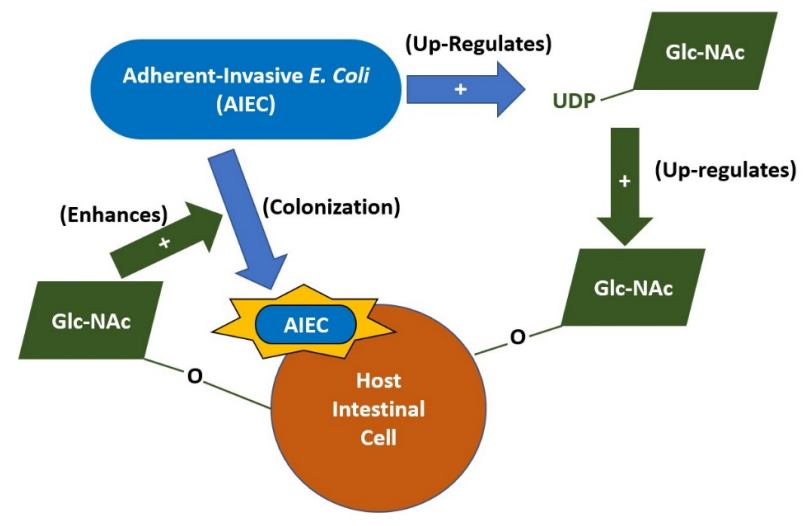

Figure 3: AIEC Up-regulates O-GlcNacs on Host Intestinal Cells to Facilitate Colonization.

AIEC (Blue) increases local UDP-GIcNAc (Green) concentration, thereby upregulating O-GIcNAcylation of host cells (Orange) and enhancing its own colonization of the $\mathrm{GI}$ mucosa. This has been shown to be a central mechanism in AlEC-induced Crohn's Disease [31]. Diagram not drawn to actual scale.

Conversely, the manipulation of glycosylation profile could potentially be performed for therapeutic or prophylactic purposes in the future.

Activating the full potential of the human immune system is a dream for clinicians and patients combatting autoimmune and infectious diseases. The understanding of how to control glycosylation patterns within the immune system could be powerful tool in realizing this dream in the years to come.

\section{Recognizing Glycosylation Enhanced Diagnostics}

Early diagnosis of diseases is just as important as having an effective therapeutic option. The earlier the detection, the more time and options that a clinician has, to formulate a treatment plan; it also means a healthier patient who can better withstand the disease as well as any side effects that the treatment may elicit. Certain glycosylation patterns are often unique to disease processes and may serve as valuable markers for early detection and diagnosis.

Many neoplasms have been discovered to have signature glycosylation patterns that may be used for early screening [32]. For example, the O-glycans in breast, colorectal, and pancreatic cancers are all aberrant from normal cells, yet are also sufficiently different from one another, allowing the potential for clinical distinction [33]. One specific example can be drawn from a subtype of pancreatic cancer, where the loss of GCNT3, a glycotransferase, leads to decreased invasion and metastasis [34]. Detect of GCNT3 in pancreatic cancer will also allow clinicians to use Talniflumate, a GCNT3 inhibitor, as a specific chemotherapy agent. In gastric cancer, it is the presence of aberrant glycosylation on serum haptoglobins that may alert clinicians to early progression of the neoplasm [35]. And finally, in lung adenocarcinoma, a striking increase in paucimannose and high mannose glycans was observed [36].

In addition to cancer, glycosylation pattern changes were observed in many other inflammatory diseases, such as viral bronchitis, COPD, and oral mucositis [37,38,39]. Interestingly, glycosylation profiles have also been explored as a marker for male infertility [40]. Clusterin, a crucial protein component of semen, plays an essential part in the capacitation of sperm and its overall survival in the uterus and fallopian tube. Analysis of six different $\mathrm{N}$-glycosylation sites on clusterin shows differential patterns that may be linked with male infertility, though further studies are needed [41].

Studying glycosylation patterns may leads to earlier diagnosis of cancer and other diseases, potentially leading to more treatment options and improved prognosis.

\section{Applying Glycosylation to Covid-19 Research}

In December 2019, a regional outbreak of SARS-Cov-2 in China rapidly developed into a global pandemic, infecting over 8 million people across 213 countries and regions, causing over 450,000 deaths by June 2020 [42,43]. Researchers are working tirelessly to develop effective therapeutics and eventually, a vaccine that could be delivered globally $[44,45]$. Glycosylation is one such technique that they have employed in their quest to battle this pandemic.

Researchers started by carefully analyzing the glycosylation pattern differences between SARS-CoV-2 and the original SARS-CoV. While the former had a $83.9 \%$ similarity in Cryo-EM analysis of receptor binding domain with the latter, differences were observed in the form of a new glycosylation site [46]. This crucial difference may help explain SARS-CoV-2's antigenic variability and its differences in transmission dynamics. Further studies performed on the SARS-CoV-2 Spike (S) protein revealed extensive glycan shielding of surface sites, hindering potential antibodies from binding [47]. Glycans only making up $17 \%$ of the total S protein molecular weight; however, $42 \%$ of all glycans are concentrated on the protein surface, contributing to shielding. See Figure 4 for a simplified depiction of this finding. Circumventing these shielding glycans will be a great hurdle in developing antibodies to provide temporary, passive immunity. 


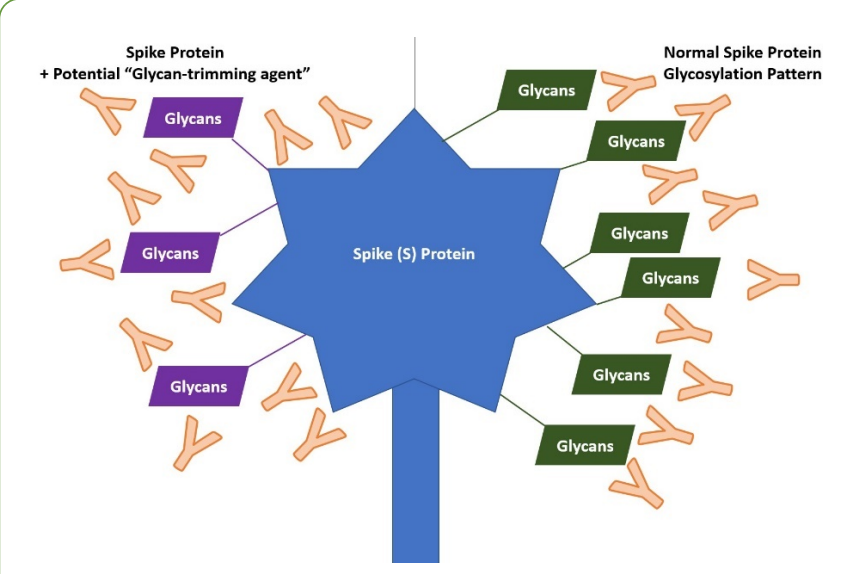

Figure 4: Glycan-shielding of SARS-CoV-2 Spike (S) Protein Hinders Antibody Interaction.

On the right half the diagram, the SARS-CoV-2 S Protein (Blue) is coated with abundant glycans (Green), thus occluding antibody (Orange) recognition and binding sites. On the left half of the diagram, the S Protein is treated with a potential "glycan-trimming" agent, reducing the shielding effect. The remaining glycans (Purple) are present but sparse, allowing more antibodies to effectively bind to epitopes. Other methods of circumventing glycan shielding should also be explored [47]. Diagram is not drawn to actual scale.

Discussions and efforts are also being made to enhance the body's endogenous anti-viral response via manipulating antiglycan antibodies [48]. Some papers have also described the relative increased resistance of human blood group 0 to infection with SARS-CoV-2, as observed in exposed. healthcare workers [49]. This difference may be due to differential glycosylation patterns on human blood groups as well as the effects of anti-A isoagglutinin in blocking viral entry. Future research is still needed to analyse and ascertain these promising findings to apply them to therapeutic use.

\section{Concluding Remarks}

The rapid advancement in laboratory techniques over the recent decades have allowed researchers to reach newer microscopic and molecular depths. This provides excellent opportunities to tap into the potential clinical uses of glycosylation, a nearly ubiquitous post-translational modification in eukaryotes [1].

The multifaceted and complex nature of glycosylation is a "double-edged" sword. On one hand, it increases the difficulty of research, observation and analysis exponentially; but on the other hand, it also allows researchers to use glycosylation in multiple outlets of clinical application. As previously mentioned, efforts have been made to conjugate existing and new pharmaceuticals to various glycans to enhance stability, safety and clinical efficacy [8]. Differential glycosylation is being profiled in diseases to be developed as novel vaccine or drug targets [25]. Attempts have been made to change glycosylation patterns of immune components to boost the body's inherent strength to fight infection and diseases [27]. Glycosylation motifs are being explored as early diagnostic markers in cancers, autoimmune and infectious diseases [32]. Furthermore, glycosylation is also applied to research for therapeutics and vaccines during the ongoing Covid-19 pandemic [46]. See Figure 5 for an abbreviated summary of the general directions in researching the clinical applications of glycosylation.

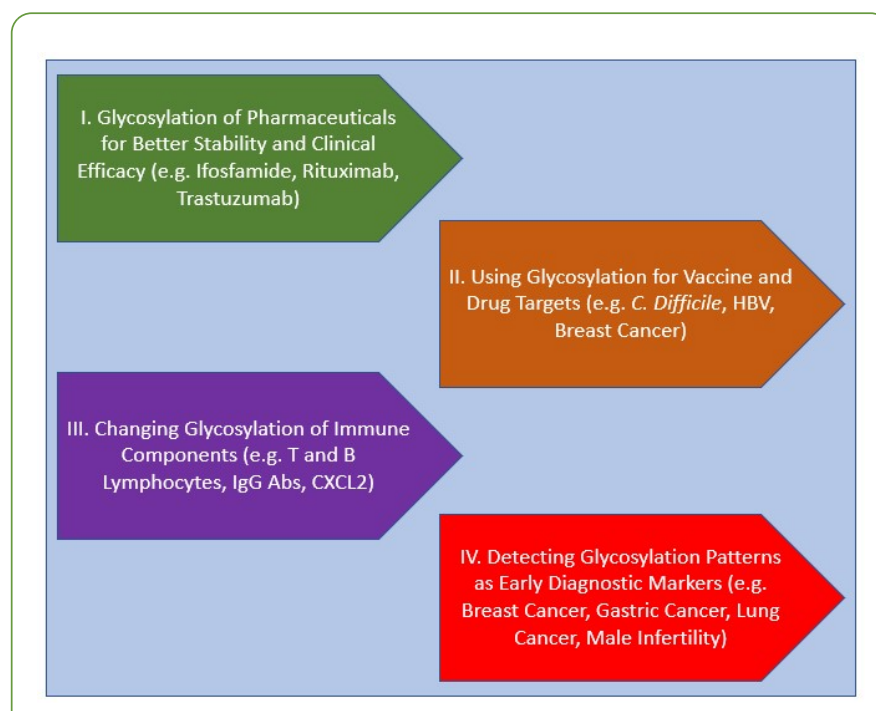

Figure 5: Brief Summary of Approaches of Research in Clinical Applications of Glycosylation.

This figure provides a quick graphic summary of different directions in the clinical potential of glycosylation. The examples are compiled from multiple studies $[8,9,10,18,20,23,26,29,32,40]$.

Looking ahead. glycosylation is in a unique position to help researchers design new methods of preventing, detecting, and treating various diseases. Further studies are still needed to ascertain many of the interesting findings thus far, but the future of glycosylation and its clinical applications is a promising one.

\section{Conflict of Interests}

The authors have no conflict of interests to declare.

\section{Acknowledgment}

We thank Elizabeth C. Holland for editing the associated figures.

\section{Author's Contribution}

Yichi Zhang and Lichun Sun, both the authors contributed equally to authorship. 


\section{Funding}

We would greatly acknowledge the supports from Shenzhen Science and Technology Program. (Grant No: KQTD20170810154011370), Xiangtan Institute of Industrial Technology Collaborative Innovation, and Xiangtan Science and Technology.

\section{References}

1. Hart GW (1992) Glycosylation. Curr Opin Cell Biol 4: 1017-1023.

2. Reily C, Stewart TJ, Renfrow MB, Novak J (2019) Glycosylation in health and disease. Nat Rev Nephrol 15: 346-366.

3. Burda P, Aebi M (1999). The dolichol pathway of $\mathrm{N}$-linked glycosylation. Biochem Biophys Acta 1426: 239-257.

4. Mikolajczyk K, Kaczmarek R, Czerwinski M (2020) How glycosylation affects glycosylation: the role of $\mathrm{N}$-glycans in glycosyltransferase activity. Glycobiol 30: 1-5.

5. Wang S, Liu S, Zhang Y, He J, Coy DH, et al. (2020) Human Serum Albumin (HSA) and Its Applications as a Drug Delivery Vehicle. Health Sci J 14: 1-8.

6. Tiwari G, Tiwari R, Sriwastawa B, Bhati L, Pandey S, et al. (2012) Drug delivery systems: An updated review. Int J Pharma Invest 2: 2-11.

7. Strebhardt K, Ullrich A (2008). Paul Ehrlich's magic bullet concept: 100 years of progress. Nat Rev Canc 8: 473-480.

8. Molejon M I, Weiz G, Breccia J D, Vaccaro MI (2020) Glycoconjugation: An approach to cancer therapeutics. World J Clin Oncol 11: 110-120.

9. Kim H Y, Park M, Kang C, Heo W, Yoon SM, et al. (2020) OGlcNAcylation of light chain serine 12 mediates rituximab production doubled by thiamet G. Bioprocess Biosyst Eng 43: 863-875.

10. Joshi S, Rathore AS (2020) Assessment of Structural and Functional Comparability of Biosimilar Products: Trastuzumab as a Case Study. Bio Drugs 34: 209-223.

11. Arruda EL, Japiassu KB, de Melo Souza PL, Araujo KCF, Thomaz DV, et al. (2020) Zidovudine Glycosylation by Filamentous Fungi Leads to Better Redox Stability and Improved Cytotoxicity on B16F10 Murine Melanoma Cells. Anticancer Agents Med Chem PMID: 32329702.

12. Bai B, Wang XF, Zhang $M, N a$ L, Zhang $X$, et al. (2020) The Nglycosylation of Equine Tetherin Affects Antiviral Activity by Regulating Its Subcellular Localization. Viruses 12: 220.

13. Grimsey E, Collis DWP, Mikut R, Hilpert K (2020) The effect of lipidation and glycosylation on short cationic antimicrobial peptides. Biochim Biophys Acta Biomembr 1862: 183195.

14. Arbo BD, Andre-Miral C, Nasre-Nasser RG, Schimith LE, Santos MG, et al. (2020) Resveratrol Derivatives as Potential Treatments for Alzheimer's and Parkinson's Disease. Front Aging Neurosci 12: 103.

15. Attallah C, Aguilar MF, Forno G, Etcheverrigaray M, Brigido MM, et al. (2020) The glycosylation of anti-rhIFN-alpha2b recombinant antibodies influences the antigen-neutralizing activity. Biotechnol Lett PMID: 32285235.

16. Hurjak B, Kovacs Z, Donczo B, Katona E, Haramura G, et al. (2020) N-glycosylation of blood coagulation factor XIII subunit B and its functional consequence. J Thromb Haemost 18: 1302-1309.

17. Lee SM, Jeong Y, Simms J, Warner ML, Poyner DR, et al. (2020) Calcitonin Receptor N-Glycosylation Enhances Peptide Hormone Affinity by Controlling Receptor Dynamics. J Mol Biol 432: 1996-2014.

18. Aubry A, Zou W, Vinogradov E, Williams D, Chen W, et al. (2020) In vitro Production and Immunogenicity of a Clostridium Difficile Spore-Specific BclA3 Glycopeptide Conjugate Vaccine. Vaccines 8: 73.

19. Cain JA, Dale AL, Sumer-Bayraktar Z, Solis N, Cordwell SJ (2020) Identifying the targets and functions of $\mathrm{N}$-linked protein glycosylation in Campylobacter jejuni. Mol Omics 32: A

20. Joe CCD, Chatterjee S, Lovrecz G, Adams TE, Thaysen-Andersen $M$, et al. (2020) Glycoengineered hepatitis $B$ virus-like particles with enhanced immunogenicity. Vaccine 38: 3892-3901.

21. Li SW, Wright $M$, Healey JF, Hutchinson JM, O'Rourke $S$, et al. (2020) Gene editing in $\mathrm{CHO}$ cells to prevent proteolysis and enhance glycosylation: Production of HIV envelope proteins as vaccine immunogens. PLoS One 15: e0233866.

22. Beckwith DM, Cudic M (2020) Tumor-associated O-glycans of MUC1: Carriers of the glyco-code and targets for cancer vaccine design. Semin Immunol 47: 101389.

23. Liu H, Ma L, Lin J, Cao B, Qu D, et al. (2020) Advances in molecular mechanisms of drugs affecting abnormal glycosylation and metastasis of breast cancer. Pharmacol Res 155: 104738.

24. Madigan JP, Robey RW, Poprawski JE, Huang $\mathrm{H}$, Clarke $\mathrm{CJ}$, et al. (2020) A role for ceramide glycosylation in resistance to oxaliplatin in colorectal cancer. Exp Cell Res 388: 111860.

25. Naseri R, Navabi SJ, Samimi Z, Mishra AP, Nigam M, et al. (2020) Targeting Glycoproteins as a therapeutic strategy for diabetes mellitus and its complications. Daru 28: 333-358.

26. Chang $\mathrm{YH}$, Weng $\mathrm{CL}$, Lin $\mathrm{KI}$ (2020) O-GlcNAcylation and its role in the immune system. J Biomed Sci 27: 57.

27. de Haas P, Hendriks W, Lefeber DJ, Cambi A (2020). Biological and Technical Challenges in Unraveling the Role of $\mathrm{N}$-Glycans in Immune Receptor Regulation. Front Chem 8: 55.

28. Kumpel BM, Saldova R, Koeleman CAM, Abrahams JL, Ederveen $A H$, et al. (2020) Anti-D monoclonal antibodies from 23 human and rodent cell lines display diverse IgG Fc-glycosylation profiles that determine their clinical efficacy. Sci Rep 10: 1464.

29. Corsiero E, Carlotti E, Jagemann L, Perretti M, Pitzalis C, et al. (2020) H and L Chain Affinity Maturation and/or Fab NGlycosylation Influence Immunoreactivity toward Neutrophil Extracellular Trap Antigens in Rheumatoid Arthritis Synovial B Cell Clones. J Immunol 204: 2374-2379.

30. Fonseca KL, Maceiras AR, Matos R, Simoes-Costa L, Sousa J, et al. (2020) Deficiency in the glycosyltransferase Gcnt1 increases susceptibility to tuberculosis through a mechanism involving neutrophils. Mucosal Immunol 277: 7

31. Denizot J, Barnich N (2020) When Adherent-invasive E. coli plays with host glycosylation: Does it open new perspectives for therapeutic strategies in Crohn's disease? E Bio Med 55: 102752.

32. Costa AF, Campos D, Reis CA, Gomes C (2020) Targeting Glycosylation: A New Road for Cancer Drug Discovery. Trends Cancer 4: 2 
33. Cervoni GE, Cheng JJ, Stackhouse KA, Heimburg-Molinaro J, Cummings RD (2020) O-glycan recognition and function in mice and human cancers. Biochem J 477: 1541-1564.

34. Gupta R, Leon F, Thompson CM, Nimmakayala R, Karmakar S, et al. (2020) Global analysis of human glycosyltransferases reveals novel targets for pancreatic cancer pathogenesis. $\mathrm{Br} \mathrm{J}$ Cancer 122: $1661-1672$.

35. Jeong S, Oh MJ, Kim U, Lee J, Kim JH, et al. (2020). Glycosylation of serum haptoglobin as a marker of gastric cancer: an overview for clinicians. Expert Rev Proteomics 17: 109-117.

36. Lattova E, Skrickova J, Hausnerova J, Frola L, Kren L, et al. (2020) $\mathrm{N}$-Glycan profiling of lung adenocarcinoma in patients at different stages of disease. Mod Pathol 33: 1146-1156.

37. Bouwman KM, Habraeken N, Laconi A, Berends AJ, Groenewoud $\mathrm{L}$, et al. (2020) N-glycosylation of infectious bronchitis virus M41 spike determines receptor specificity. J Gen Virol PMID: 32213247

38. Farkas A, Meszaros B, Szarka M, Szigeti M, Kappelmayer J, et al. (2020) Modeling of the desialylated human serum N-glycome for molecular diagnostic applications in inflammatory and malignant lung diseases. Curr Mol Med PMID: 32321401

39. Gebri E, Kovacs Z, Meszaros B, Toth F, Simon A, et al. (2020) NGlycosylation Alteration of Serum and Salivary Immunoglobulin a Is a Possible Biomarker in Oral Mucositis. J Clin Med 9: 61747.

40. Omolaoye TS, du Plessis SS (2020) Male infertility: A proximate look at the advanced glycation end products. Reprod Toxicol 93: 169-177.

41. Janiszewska E, Kratz EM (2020) Could the glycosylation analysis of seminal plasma clusterin become a novel male infertility biomarker? Mol Reprod Dev 87: 515-524.
42. Ogbunugafor $C B$, Miller-Dickson MD, Meszaros VA, Gomez LM, Murillo AL, et al. (2020). The intensity of COVID-19 outbreaks is modulated by SARS-CoV-2 free-living survival and environmental transmission. Medrxiv PMID: 32511513.

43. Zhang Y, Chun M, Lin Z, Ross J, Sun LC et al. (2020) Ninety Days in: A Comprehensive Review of the Ongoing COVID-19 Outbreak. Health Sciences Journal, 14: 1-8.

44. Mullard A (2020) COVID-19 vaccine development pipeline gears up. Lancet 395: 1751-1752.

45. Wang S, Zhang Y, Liu S, Peng H, Mackey V, et al. (2020) Coronaviruses and the Associated Potential Therapeutics for the Viral Infections. J Infect Dis Thear 8: 1-8.

46. Kumar S, Maurya VK, Prasad AK, Bhatt MLB, Saxena SK (2020) Structural, glycosylation and antigenic variation between 2019 novel coronavirus (2019-nCoV) and SARS coronavirus (SARSCoV). Virus Dis 31: 13-21.

47. Grant OC, Montgomery D, Ito K, Woods RJ (2020) Analysis of the SARS-CoV-2 spike protein glycan shield: implications for immune recognition. Biorxiv PMID: 32511307

48. Breiman A, Ruven-Clouet N, Le Pendu J (2020) Harnessing the natural anti-glycan immune response to limit the transmission of enveloped viruses such as SARS-CoV-2. PLoS Pathog 16: e1008556.

49. Daniele $F$ (2020) Anti-A Isohemagglutinin titers and SARS-CoV2 neutralization: implications for children and convalescent plasma selection. Br J Haematol PMID: 32516462. 